\title{
Desempenho operacional de derriçadores mecânicos portáteis, em diferentes condições de lavouras cafeeiras
}

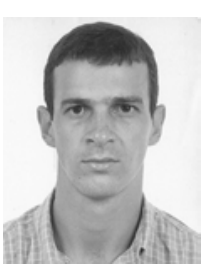

Jackson A. Barbosa ${ }^{1}$, Nilson Salvador ${ }^{2}$ \& Fábio M. da Silva ${ }^{2}$

\begin{abstract}
${ }^{1}$ Departamento de Agronomia, FAFEID, Diamantina, MG. Fone: (38) 3531-1811. E-mail: jackson_barbosa@hotmail.com (Foto) 2 DEA/UFLA. CEP 37200-000, Lavras, MG, E-mail: salvador@ufla.br; fmsilva@ufla.br
\end{abstract}

Protocolo 86 - 9/5/2003 - Aprovado em 31/4/2004

\begin{abstract}
Resumo: Dentre as operações realizadas na colheita do café, a de derriça é considerada a mais complexa, por sua grande influência no custo de colheita, sendo que a mecanização da operação de derriça do café pode ter efeito significativo em redução do custo final da saca de café colhido, além de ser na região do sul de Minas Gerais, um dos sistemas mais utilizados por produtores de pequeno e médio portes, que utilizam, nesta operação, o sistema de derriçadores mecânicos portáteis, o qual pode apresentar rendimento até oito vezes superior ao da colheita manual. Este trabalho teve como objetivo avaliar o desempenho operacional de derriçadores mecânicos portáteis utilizados na colheita de café e avaliar o custo operacional do sistema de colheita semi-mecanizado, comparativamente ao sistema de colheita manual. Conclui-se que a colheita de café semi-mecanizada apresentou desempenho operacional superior ao da colheita manual, tornando-se uma alternativa viável para os produtores de pequeno a médio porte, visando minimizar os custos de colheita.
\end{abstract}

Palavras-chave: colheita de café, derriçadores mecânicos portáteis

\section{Operational performance of portable mechanical coffee harvesters in different conditions of coffee planting}

\begin{abstract}
In the coffee harvesting, the harvest is considered the most complex operation because of its great influence in the harvesting cost therefore, mechanization of the same may have significant effect on reduction of the final cost of the bag of harvested coffee. The objective of this research was to evaluate the operational performance of portable coffee harvesting machines and to evaluate the operational cost of the semi-mechanized harvest system in comparison to the system of manual harvest. The trials were conducted in five plantation of coffee crop variety Catuaí 74 (IAC 62), under different management conditions. Three models of portable coffee harvesting machines available commercially and in use were evaluated. The experiment was constituted of 20 treatments (combinations of three types of portable coffee harvesting machines plus manual harvest and five types of coffee crop), in a randomized block design. On the basis of obtained results, it may be concluded that the semi-mechanized coffee harvester presented superior operational performance in comparison to manual harvest system, becoming a viable alternative for the small to medium size farmers in order to minimize the production costs.
\end{abstract}

Key words: coffee harvester, portable coffee harvesting machine

\section{INTRODUÇÃO}

Dentre as operações realizadas na colheita do café, destacase a de derriça, considerada a mais complexa, sobretudo pela influência no custo de colheita (Matiello \& Pinto, 1998), sendo que a mecanização da operação de derriça do café pode ter efeito significativo em redução no custo final da saca de café colhido (Inamasu \& Andrade, 1997; Silva \& Salvador, 1998; Silva et al., 1998 ).

Estudos referentes ao processo de colheita mecanizada de produtos agrícolas tiveram origem no início da década de setenta, baseados no método de vibração, e as primeiras colhedoras mecânicas de café apareceram na década de oitenta (Fava et al., 1979).

Na região do sul de Minas Gerais, um dos sistemas que mais têm sido utilizados por produtores de pequeno e médio porte na mecanização da operação de derriça do café, é o uso de derriçadores mecânicos portáteis, os quais, segundo Silva et al. (1997), apresentam rendimento até oito vezes superior ao da colheita manual.

Barros et al. (1995) realizaram testes através da derriçadora mecânica portátil de café Agromática e mostraram que o 
equipamento apresentou rendimento oito vezes superior ao da operação de derriça manual. Ao se medir a desfolha dos pés de café, observou-se que a mesma foi semelhante para ambas as modalidades porém quebrou quatro vezes mais ramos primários e secundários que na colheita manual e o repasse de café necessário foi da ordem de $10 \%$ na operação de derriça mecânica.

Avaliando aspectos econômicos e o rendimento operacional de uma derriçadora mecânica portátil de origem italiana, Favarin et al. (1998) constataram que o equipamento sofreu variação de rendimento por hectare, devido a fatores como altura de plantas e estado de maturação dos frutos. A amplitude de variação foi de 0,08 ha dia $^{-1}$ a 0,28 ha dia $^{-1}$, com média de 0,17 ha dia $^{-1}$. Constatou-se economia de mão-de-obra média de $21,75 \%$ em relação à colheita manual.

Para comparar o desempenho de três sistemas de derriça, Salvador et al. (1998) utilizaram derriçadores mecânicos portáteis modelos Agromática e Shindaiwa, comparando-os com o sistema de derriça manual. Os testes foram realizados na região de Três Pontas, MG, numa lavoura de variedade Catuaí, cujas características são: 19 anos, 2,10 m de altura média, 2,03 m de diâmetro de saia, $3,40 \mathrm{~m}$ entre ruas e $0,55 \mathrm{~m}$ entre plantas na linha, carga pendente com $98 \%$ de frutos secos. Mediante as condições e a forma como foram realizados os testes e os resultados obtidos, concluiu-se que os derriçadores Shindaiwa e Agromática apresentam desempenho semelhante; a produtividade dos derriçadores superou a da derriça manual, em cerca de três vezes e que os danos à planta na derriça manual foram menores, tendo em vista que a mesma é seletiva.

Para avaliar os possíveis reflexos da colheita mecanizada à planta em relação à safra futura, prosseguiu-se um ensaio de colheita de parcelas colhidas mecanicamente no ano anterior, por Garcia \& Fioravante (2002), no ano agrícola de 2002. Pela análise das produções obtidas observou-se que não houve prejuízos na safra seguinte.

Com o objetivo de avaliar a influência do sistema de colheita na qualidade do café realizou-se, com base em Borém et al. (2002) um experimento no município de Campos Gerais, MG, no qual foram estudados seis sistemas de colheita: a) derriça manual no pano, com recolhimento e abanação manual; b) derriça manual no chão, com recolhimento e abanação manual; c) derriça mecanizada no pano, com derriçador mecânico portátil, com recolhimento e abanação manual; d) derriça mecanizada no chão, com derriçador mecânico portátil, com recolhimento e abanação manual; e) derriça mecanizada no chão, com derriçador mecânico portátil, com recolhimento e abanação mecanizados; e, f) derriça mecanizada com derriçadora automotriz. Após a secagem o café foi beneficiado e realizadas as seguintes análises: condutividade elétrica, lixiviação de potássio e prova de xícara. Os valores médios de condutividade elétrica e lixiviação de potássio não apresentaram diferenças significativas em função dos sistemas estudados. Quanto à prova de xícara, não houve tendência definida, em função dos sistemas de colheita.

Com este trabalho objetivou-se avaliar o desempenho operacional de derriçadores mecânicos portáteis utilizados na colheita de café, na região de Lavras, $\mathrm{MG}$, e o custo operacional do sistema de colheita semi-mecanizado, comparativamente com o sistema de colheita manual.

\section{MATERIAL E MÉTODOS}

\section{Lavouras}

Os testes foram conduzidos em cinco lavouras da variedade Catuaí 74 (IAC 62), localizadas na região de Lavras, MG, no período de junho a setembro de 2001, estando as lavouras sob diferentes condições de manejo, citando-se:

a) cinco anos de implantação, composto por um ramo ortotrópico, produtividade de $4,22 \mathrm{~L}_{\text {planta }}{ }^{-1}, 12,70 \%$ de café verde (valor médio do estande), espaçamento de 3,5 x 0,7 m, altura média das plantas de $1,7 \mathrm{~m}$, diâmetro médio da saia de 1,8 $\mathrm{m}$ e diâmetro médio do ramo ortotrópico de $32 \mathrm{~mm}$, denominada lavoura 1 ;

b) três anos após recepa, formado por três ramos ortotrópicos, produtividade de $5,17 \mathrm{~L} \mathrm{planta}^{-1}, 41,90 \%$ de café verde (valor médio do estande), espaçamento: $3,5 \times 0,8 \mathrm{~m}$, altura média das plantas de $1,8 \mathrm{~m}$, diâmetro médio da saia de $1,8 \mathrm{~m}$, diâmetro médio dos ramos ortotrópicos de $24 \mathrm{~mm}$, denominada lavoura 2;

c) três anos após recepa, duas plantas por cova, produtividade de 3,79 $\mathrm{L}_{\text {planta }}{ }^{-1}, 6,37 \%$ de café verde (valor médio do estande), espaçamento de $3,0 \times 1,0 \mathrm{~m}$, altura média das plantas de 1,3 m, diâmetro médio da saia de $1,3 \mathrm{~m}$, diâmetro médio dos ramos ortotrópicos de $22 \mathrm{~mm}$, denominada lavoura 3;

d) três anos de implantação, composto por um ramo ortotrópico, irrigado por pivô central com lâmina d'água controlada, produtividade de $4,11 \mathrm{~L}_{\text {planta }}{ }^{-1}, 28,42 \%$ de café verde (valor médio do estande), espaçamento de 3,5 x 0,8 $\mathrm{m}$, altura média das plantas de $1,2 \mathrm{~m}$, diâmetro médio da saia de $1,41 \mathrm{~m}$, diâmetro médio do ramo ortotrópico de $28 \mathrm{~mm}$, denominada lavoura 4;

e) vinte e dois anos de implantação, bem enfolhado, com média de quatro ramos ortotrópicos por planta, produtividade de 4,92 $\mathrm{L} \mathrm{planta}^{-1}, 25,86 \%$ de café verde (valor médio do estande), espaçamento de 3,5 x 0,6 m, altura média das plantas de 2,20 m, diâmetro médio da saia de 2,20 m, diâmetro médio dos ramos ortotrópicos de $36 \mathrm{~mm}$, denominada lavoura 5 .

\section{Derriçadores mecânicos portáteis}

Avaliaram-se três modelos de derriçadores mecânicos portáteis disponíveis comercialmente e em uso contínuo por produtores da região, denominados derriçadores modelos 1,2 e 3. Todos os modelos são constituídos por motor de combustão interna. As características operacionais dos derriçadores são apresentadas na Tabela 1 .

Determinação dos custos de utilização dos derriçadores mecânicos portáteis: $O$ custo de utilização de uma máquina agrícola é dividido em custos fixos e custos variáveis. Os custos

Tabela 1. Características operacionais dos derriçadores mecânicos portáteis

\begin{tabular}{cccc} 
Modelo & $\begin{array}{c}\text { Potência do Motor } \\
(\mathrm{kW})\end{array}$ & $\begin{array}{c}\text { Amplitude } \\
(\mathrm{mm})\end{array}$ & $\begin{array}{c}\text { Freqüência } \\
(\mathrm{Hz})\end{array}$ \\
\hline 1 & 0,735 & 180 & 18,33 \\
2 & 0,882 & 15 & 63,90 \\
3 & 0,882 & 12 & 50,05 \\
\hline
\end{tabular}


fixos envolvem aqueles que não variam com a intensidade de uso da máquina, citando-se a depreciação, o juro sobre o capital investido, os impostos, o custo associado ao abrigo da máquina e seguro, enquanto os custos variáveis são influenciados pela intensidade de uso da máquina e envolvem os custos associados a combustíveis e lubrificantes, custos de reparo e manutenção e custos da mão-de-obra para operação da máquina (Balastreire, 1987). Para determinação do custo do uso dos derriçadores mecânicos portáteis utilizaram-se as seguintes metodologias: depreciação linear para vida útil de 2.500 horas, taxa de juros de $8,75 \%$ a.a., taxa de abrigo e seguro de $2 \%$ a.a., consumo de combustível e óleo lubrificante, segundo medidas de campo, reparo e manutenção, de acordo com o plano de manutenção do manual dos fabricantes e salário do operador de acordo com a produtividade, sendo de $\mathrm{R} \$ 2,50$ por medida de $60 \mathrm{~L}$.

\section{Delineamento estatístico}

O experimento constituiu-se de 20 tratamentos delineados em blocos ao acaso, com 8 repetições. Os tratamentos resultaram das combinações entre os 3 tipos de derriçadores mecânicos portáteis, comparados ao sistema de colheita manual, e os 5 tipos de lavoura.

As variáveis avaliadas foram: volume de café derriçado por unidade de tempo, volume de café colhido por unidade de tempo e custo da operação de colheita. Todas as variáveis foram comparadas pelo teste de Tukey a nível de $5 \%$ de significância.

Uma vez que a equipe do derriçador mecânico portátil se compõe de 3 funcionários, um dos quais operador e 2 repassadores/levantadores de café, a equipe do sistema manual também constou de 3 funcionários.

Os derriçadores mecânicos portáteis foram comparados entre si e entre o sistema de derriça manual.

\section{RESULTADOS E DISCUSSÃO}

$\mathrm{Na}$ Tabela 2 verifica-se que tanto o tipo de lavoura quanto o sistema de colheita exerceram efeitos significativos sobre o volume de café derriçado, e, ainda, que as lavouras denominadas 4 e 3 proporcionaram os maiores valores, enquanto a lavoura denominada 5 resultou no menor valor. As lavouras 1 e 2 mantiveram-se em posição intermediária. O menor valor de volume de café derriçado por unidade de tempo da lavoura 5 em relação às lavouras 4 e 3 , deve ser atribuído, em grande parte, à maior densidade de ramos desse tipo de lavoura.

Os sistemas de colheita mecanizada apresentaram diferença significativa comparativamente ao sistema manual, com maior valor atribuído aos modelos 2 e 3 , cujo efeito se deve à maior freqüência dos mecanismos de colheita, a qual atinge os níveis definidos para colheita de grãos desde o estádio verde até o seco (Filgueiras, 2000). O volume de café derriçado pela equipe mecanizada foi, em média, $251 \%$ superior ao volume de café derriçado pela equipe manual.

Os resultados do volume de café efetivamente colhido (derriçado, levantado e ensacado) são apresentados na Tabela 2. Verifica-se que o tipo de lavoura não influenciou no volume de café efetivamente colhido, porém os sistemas de colheita mecanizada exerceram efeitos significativos sobre essa variável,
Tabela 2. Volume de café derriçado $\left(\mathrm{L} \mathrm{h}^{-1}\right)$, efetivamente colhido $\left(\mathrm{L} \mathrm{h}^{-1}\right)$ e custo da operação de colheita (R \$ por medida de $60 \mathrm{~L}$ ) em função de cinco tipos de lavoura e de quatro sistemas de colheita*

\begin{tabular}{|c|c|c|c|c|c|}
\hline \multirow[b]{2}{*}{ Lavoura } & \multirow[b]{2}{*}{ Manual } & \multicolumn{3}{|c|}{ Sistema de Colheita - Modelo } & \multirow[b]{2}{*}{ Média } \\
\hline & & 1 & 2 & 3 & \\
\hline \multicolumn{6}{|c|}{ Café derriçado $-\mathrm{L} \mathrm{h}^{-1}$} \\
\hline 1 & 74 & 125 & 207 & 181 & $146,75 \mathrm{~b}$ \\
\hline 2 & 90 & 155 & 274 & 219 & $184,50 \mathrm{~b}$ \\
\hline 3 & 62 & 233 & 440 & 351 & $271,50 \mathrm{a}$ \\
\hline 4 & 67 & 241 & 336 & 326 & $242,50 \mathrm{a}$ \\
\hline 5 & 56 & 130 & 179 & 152 & $129,25 \mathrm{c}$ \\
\hline $\begin{array}{c}\text { Média } \\
\mathrm{CV}=8,08 \%\end{array}$ & $69,80 \mathrm{C}$ & $176,80 \mathrm{~B}$ & $287,20 \mathrm{~A}$ & $245,80 \mathrm{~A}$ & 194,90 \\
\hline
\end{tabular}

\begin{tabular}{|c|c|c|c|c|c|}
\hline \multicolumn{6}{|c|}{ Café efetivamente colhido $-\mathrm{L} \mathrm{h}^{-1}$} \\
\hline 1 & 74 & 104 & 114 & 110 & $100,50 \mathrm{a}$ \\
\hline 2 & 90 & 131 & 131 & 129 & $120,75 \mathrm{a}$ \\
\hline 3 & 62 & 129 & 156 & 136 & $120,75 \mathrm{a}$ \\
\hline 4 & 67 & 127 & 131 & 178 & $125,75 \mathrm{a}$ \\
\hline 5 & 56 & 75 & 99 & 97 & $81,75 \mathrm{a}$ \\
\hline $\begin{array}{c}\text { Média } \\
\mathrm{CV}=14,70 \%\end{array}$ & $69,80 \mathrm{~B}$ & $113,20 \mathrm{~A}$ & $126,20 \mathrm{~A}$ & $130 \mathrm{~A}$ & 109,80 \\
\hline \multicolumn{6}{|c|}{ Custo da operação de colheita $-\mathrm{R} \$$ por $60 \mathrm{~L}$} \\
\hline 1 & 6,83 & 6,14 & 6,18 & 6,20 & $6,34 \mathrm{~b}$ \\
\hline 2 & 5,60 & 4,94 & 5,65 & 6,04 & $5,56 \mathrm{~b}$ \\
\hline 3 & 8,13 & 5,17 & 4,68 & 5,12 & $5,78 \mathrm{~b}$ \\
\hline 4 & 7,52 & 4,83 & 5,52 & 3,86 & $5,43 \mathrm{~b}$ \\
\hline 5 & 9,00 & 8,57 & 7,02 & 7,24 & $7,96 \mathrm{a}$ \\
\hline $\begin{array}{c}\text { Média } \\
\mathrm{CV}=4,86 \%\end{array}$ & $7,42 \mathrm{~A}$ & 5,93 B & $5,81 \mathrm{~B}$ & $5,69 \mathrm{~B}$ & 6,21 \\
\hline
\end{tabular}

si a $5 \%$ de probabilidade pelo teste de Tukey.

ao serem comparados com o sistema manual, não apresentando diferença estatística entre si. Ao se comparar o volume de café efetivamente colhido, observa-se que o derriçador mecânico portátil apresenta rendimento $64 \%$ superior aos funcionários com função de repasse e levante, indicando a necessidade de otimizar o número de funcionários, uma vez que os derriçadores têm capacidade operacional superior.

A lavoura denominada 5 apresentou o maior custo de colheita, diferindo significativamente das demais, as quais não diferiram entre si (Tabela 2). O sistema manual mostrou custo operacional superior ao sistema mecanizado, independente do modelo de derriçador mecânico utilizado, concordando com as afirmações de Inamasu \& Andrade (1997), Matielli \& Pinto (1998), Silva \& Salvador (1998) e Silva et al. (1998). Em média, o custo da colheita manual foi $28 \%$ superior ao custo da colheita mecanizada.

\section{CONCLUSÕES}

1. O sistema de derriça de café mecanizado apresentou desempenho operacional superior ao sistema de derriça de café manual, com melhores resultados atribuídos aos derriçadores com freqüência superior a $50 \mathrm{~Hz}$, e lavouras com menor densidade de ramos.

2. O número de funcionários envolvidos na operação de levante e repasse deve ser otimizado para maximizar a eficiência do sistema de colheita mecanizado.

3. O sistema de colheita mecanizado apresentou menor custo operacional, comparativamente ao sistema de colheita manual. 


\section{LITERATURA CITADA}

Balastreire, L.A. Máquinas agrícolas, São Paulo: Manole, 1987, $307 \mathrm{p}$.

Barros, U.V.; Barbosa, C.M.; Matiello, J.B. Testes com derriçadora manual Agromática na colheita do café. In: Congresso Brasileiro de Pesquisas Cafeeiras, 21, 1995, Rio de Janeiro. Resumos... Rio de Janeiro: MAA/PROCAFÉ, 1995. p.124-125.

Borém, F.M.; Carvalho Junior, C.; Pereira, R.G.F.A.; Silva, V.A. Influência de diferentes sistemas de colheita na qualidade do café (Coffe arabica L.). In: Congresso Brasileiro de Pesquisas Cafeeiras, 28, 2002, Caxambú. Resumos... Rio de Janeiro: MAPA/PROCAFÉ, 2002. p.400-401.

Fava, J.F.M.; Honda, A.I.; Satori, S.; Bastos, M.V. Eficiência da colheita mecânica nas variedades Mundo Novo e Catuaí em diferentes condições de lavoura. In: Congresso Brasileiro de Pesquisas Cafeeiras, 5, 1979, Araxá. Resumos... Rio de Janeiro: IBC, 1979. p.267-272.

Favarin, J.L.; Loyola, L.M.F.; Romero, J.C.P. Avaliação dos aspectos econômicos e rendimento operacional da colheita pneumática em relação à colheita manual. In: Congresso Brasileiro de Pesquisas Cafeeiras, 24, 1998, Poços de Caldas. Resumos... Rio de Janeiro: SDR/PROCAFÉ/PNFC, 1998. p.175-176.

Filgueiras, W.H.; Queiroz, D.M.; Dias, G.P.; Della Lucia, R.M. Propriedades mecânicas da madeira do café. In: Simpósio de Pesquisa dos Cafés do Brasil, 1, 2000, Poços de Caldas. Resumos expandidos...Brasília: Embrapa Café e MINASPLAN, 2000. p.1081-1084.
Garcia, A.W.R.; Fioravante, N. Efeito do uso de derriçadoras de café portáteis na produtividade do cafeeiro no ano seguinte. In: Congresso Brasileiro de Pesquisas Cafeeiras, 28, 2002, Caxambu. Resumos... Rio de Janeiro: MAPA/ PROCAFÉ, 2002.p.51.

Inamasu, R.Y.; Andrade, J.G. Teste da nova máquina para derriçar café. In: Congresso Brasileiro de Pesquisas Cafeeiras, 23, 1997, Manhuaçu, MG. Resumos... Rio de Janeiro: SDR/ PROCAFÉ/PNFC, 1997.p.12-14.

Matiello, J.B.; Pinto, J.F. Comparativo de rendimento em diversos processos de colheita manual de café. In: Congresso Brasileiro de Pesquisas Cafeeiras, Poços de Caldas, 24, 1998. Resumos... Rio de Janeiro: SDR/PROCAFÉ/PNFC, 1998. p.1314.

Salvador, N.; Silva, F.M.; Bomfim, I.P.; Granato, L.R.N. Estudo comparativo de sistemas de derriça de café. In: Congresso Brasileiro de Pesquisas Cafeeiras, 24, 1998, Poços de Caldas. Resumos... Rio de Janeiro: SDR/PROCAFÉ/PNFC, 1998 p.227-228.

Silva, F.M.; Carvalho, G.R.; Salvador, N. Mecanização da colheita do café. Informe Agropecuário, Belo Horizonte, v.18, n.187, p.43-54, 1997.

Silva, F. M.; Salvador, N. Mecanização da lavoura cafeeira. Lavras; Ed. Universidade Federal de Lavras. 1998. 55p

Silva, F.M.; Salvador, N.; Barbosa, R.R.; Abreu, E.M. Desempenho da operação mecanizada de derriça do café. Revista Engenharia na Agricultura, Viçosa, v.6, n.2, p.74-80, 1998. 